ANNALS OF

CLINICAL

NEUROPHYSIOLOGY

\title{
Uncommon coexistence of myasthenia gravis and amyotrophic lateral sclerosis
}

\author{
Eun Bin Cho ${ }^{1,2}$, Tae-won Yang ${ }^{1}$, Heejeong Jeong ${ }^{1}$, Changhyo Yoon ${ }^{1}$, Seunguk Jung ${ }^{1,2}$, and \\ Ki-Jong Park ${ }^{1,2}$ \\ 'Department of Neurology, Gyeongsang National University Changwon Hospital, Changwon, Korea \\ ${ }^{2}$ Department of Neurology, Gyeongsang Institute of Health Science, College of Medicine, Gyeongsang National \\ University, Jinju, Korea
}

Received: June 7, 2019

Revised: June 23, 2019

Accepted: July 8, 2019

\section{Correspondence to}

\section{Ki-Jong Park}

Department of Neurology, Gyeongsang National University Changwon Hospital, 11 Samjeongja-ro, Seongsan-gu, Changwon 51472, Korea

Tel: +82-55-214-3810

Fax: +82-55-214-2638

E-mail:pkjong@gnu.ac.kr

\section{ORCID}

\section{Eun Bin Cho}

https://orcid.org/0000-0003-2021-8587

Tae-won Yang

https://orcid.org/0000-0002-8113-2384

Heejeong Jeong

https://orcid.org/0000-0001-6531-1860

Changhyo Yoon

https://orcid.org/0000-0002-8875-6333

Seunguk Jung

https://orcid.org/0000-0003-2331-024X

\section{Ki-Jong Park}

https://orcid.org/0000-0003-4391-6265

Myasthenia gravis (MG) and amyotrophic lateral sclerosis (ALS) are distinct disorders. ALS affects motor neurons that control muscle movement, while MG controls communication between neurons and muscles, which occurs at neuromuscular junctions. However, on rare occasions, ALS develops after MG and vice versa. The coexistence of the two diseases represents a diagnostic challenge and requires thoughtful interpretation of clinical features. We present the case of a 53-year-old Korean male who developed ALS after MG, confirmed by clinical and electrophysiological follow-up.

Key words: Myasthenia gravis; Amyotrophic lateral sclerosis; Overlap

Myasthenia gravis (MG) is an autoimmune disorder of the neuromuscular junction characterized by fluctuating muscle weakness and fatigability. By contrast, amyotrophic lateral sclerosis (ALS) is a progressive neurodegenerative disorder affecting motor neurons in the brain and the spinal cord. MG and ALS are rare diseases, with incidence rates of 2.40/100,000 and 1.20/100,000, respectively, in South Korea. ${ }^{1,2}$ However, the co-occurrence of MG and ALS has been described in a few reports, suggesting a possible association of the two conditions. ${ }^{3-5}$ There is also evidence that several autoimmune disorders, including MG, are associated with a small but increased risk for ALS. ${ }^{6}$ A recent study reported that $0.75 \%$ of patients with ALS were also affected by MG, a number that is higher than expected. ${ }^{5}$ Here, we report the case of a patient with seropositive ocular MG who later developed ALS.

\section{CASE}

A 53-year-old male presented with ptosis and intermittent diplopia in May 2016. According to his medical history, he underwent thymectomy and postoperative radiotherapy

This is an Open Access article distributed under the terms of the Creative Commons Attribution Non-Commercial License /http:// creativecommons.org/licenses/by-nc/4.0) which permits unrestricted non-commercial use, distribution, and reproduction in any medium, provided the original work is properly cited. 
for thymoma in May 2011 and had been diagnosed with ocular MG in September 2012. He had no family history of neuromuscular disorders. He had been symptom-free on pyridostigmine and had therefore stopped the medicine 3 months before his relapse of ocular symptoms. On neurological examination, he had spontaneous bilateral ptosis and fatigable diplopia, but there were no symptoms or signs of weakness in the bulbar, limb, or respiratory muscles. Deep tendon reflexes (DTRs) were normoactive in four limbs, and Babinski signs were absent. Acetylcholine receptor antibodies (AChR-ab level = $11.083 \mathrm{nmol} / \mathrm{L}$ ) and anti-titin antibodies were positive, and the result of the repetitive nerve stimulation test (RNST) was normal. We prescribed pyridostigmine, and 3 months later he arbitrarily stopped the medication without ocular symptoms. In October 2016, he started to experience exertional dyspnea and orthopnea without ocular symptoms and visited our pulmonary medicine department in November. Pulmonary function testing revealed restrictive ventilator function with reduced forced vital capacity (FVC; $2.87 \mathrm{~L}$ and 62\%). His dyspnea slowly worsened without diurnal variation, and he came back to our clinic in March 2017. On neurological examination, he showed slurred speech and weakness in the proximal left arm (4/5 using the Medical Research Council grading system); these symptoms did not show diurnal variation. Sensory evaluation was normal. DTRs were decreased in the left arm but normoactive in the right arm and both legs. Hoffmann's reflex and Babinski signs were absent. The patient's AChR-ab level (14.489 nmol/L) was mildly elevated compared to before, but the RNST was again normal. Suspecting generalization of MG, we prescribed oral prednisolone, pyridostigmine, and azathioprine. However, his overall symptoms slowly progressed, and his right arm, face, and neck weakened. Generalized muscle fasciculation including the tongue, hand tremors, and leg cramps appeared, and the atrophy of the tongue and the left arm became prominent. In June 2017, a nerve conduction study was performed and revealed decreased compound motor action potential amplitudes in the left axillary and musculocutaneous nerves. Electromyography (EMG) showed fibrillation potentials and positive sharp waves with chronic denervation in the left first dorsal interosseous, triceps brachii, biceps brachii, and thoracic paraspinal muscles and chronic denervation in the bulbar and lumbosacral regions; fasciculation potentials were also observed in the left biceps muscle. Biochemical investigations, his thyroid profile, tumor markers, and paraneoplastic antibodies other than anti-titin antibodies were normal. A genetic test for Kennedy's disease was negative. Computed tomography (CT) scans of the chest and brain did not reveal remarkable abnormalities. Spine CT showed no evidence of cord or root compression. The patient's FVC (1.77 $L$ and 37\%) was further reduced, and his maximal inspiratory pressure (MIP) and maximal expiratory pressure (MEP) were also decreased, at $23 \mathrm{cmH}_{2} \mathrm{O}$ (normal, >46) and $43 \mathrm{cmH}_{2} \mathrm{O}$ (normal, >72), respectively. He arbitrarily stopped the medication again, because of the lack of clinical improvement. In September 2017, he visited the emergency room of our institution, with acute hypercapnic respiratory failure after taking a sleeping pill. Weaning from mechanical ventilation was possible; however, he needed bilevel positive airway pressure (BiPAP) support while lying down or sleeping. In spite of intravenous immunoglobulin (IVIG; 0.4 $\mathrm{g} / \mathrm{kg}$ for 5 days) and high-dose steroid treatment, dysphagia and leg weakness appeared, and his overall symptoms further progressed (Fig. 1). During a follow-up, hyperactive knee jerks were observed. He was then diagnosed with clinically probable laboratory-supported ALS, according to the revised El Escorial criteria:? upper motor neuron (UMN) signs in the lumbar region, lower motor neuron (LMN) signs in the bulbar and cervical regions, and active and chronic denervation in the cervical and thoracic regions determined by EMG.

\section{DISCUSSION}

We concluded that the patient developed ALS with stable MG, based on our clinical and neurophysiological findings: 1) chronic progressive asymmetric muscle weakness in a myotomal distribution spreading to adjacent regions without response to immune-modulating therapy; 2) generalized fasciculation and muscle atrophy usually seen in motor neuron diseases; 3) normal RNST in spite of clinical deterioration; 4) EMG findings compatible with motor neuron diseases, with widespread denervation even outside of motor deficit territories; and 5) coexistence of UMN and LMN signs. In our case, lower motor neuron deficits were found in more than two body regions (initially in cervical and thoracic regions), thus meeting the minimum requirements of a diagnosis of ALS. ${ }^{7}$ However, caution was warranted regarding an ALS 


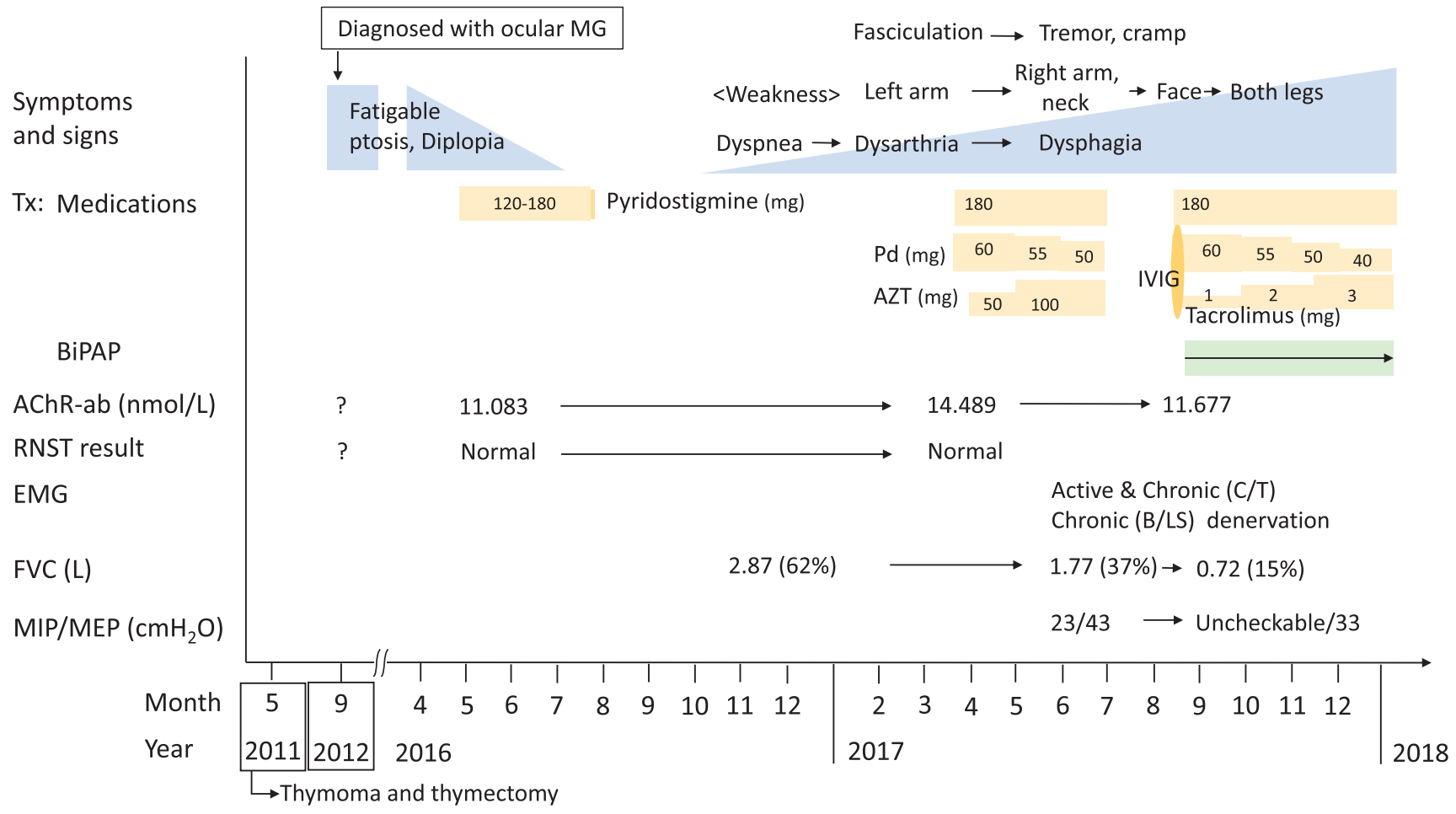

Fig. 1. Summary of the clinical course, medications, laboratory and electrophysiologic findings during the follow-up. MG, myasthenia gravis; Tx, treatment; Pd, prednisolone; AZT, azathioprine; IVIG, intravenous immunoglobulin; AChR-ab, acetylcholine receptor antibody; RNST, repetitive nerve stimulation test; FVC, forced vital capacity; BiPAP, bilevel positive airway pressure; MIP/MEP, maximal inspiratory pressure/maximal expiratory pressure; EMG, electromyography; C, cervical; T, thoracic; LS, lumbosacral; B, bulbar.

diagnosis, until the upper motor neuron sign developed during the 1.5-year follow-up. MG with muscle-specific kinase (MUSK) antibodies can mimic bulbar onset ALS, often initially showing bulbar weakness with neck and respiratory involvement and muscle atrophy. ${ }^{8}$ We did not test for MuSK antibodies; however, the coexistence of AChR and MuSK antibodies is rare, and the clinical course of our patient and his poor response to immune therapy favored ALS. ${ }^{9}$

Progressive exertional dyspnea and orthopnea could be caused by diaphragmatic weakness related to MG; however, the unusual co-occurrence with ALS caused dyspnea in this case. $^{3}$ It has been suggested that autoimmune diseases, including $M G$, are associated with a small but increased risk for ALS. ${ }^{6}$ The possible links between MG and ALS may be represented by immunological dysfunction and shared autoantibodies against neuromuscular junction proteins including low-density lipoprotein receptor-related protein $4 .{ }^{10}$ In a previous review of 12 patients with ALS after MG, males were affected twice as often as females, which might reflect a higher incidence of ALS in the male population. ${ }^{4}$ Myasthenic symptoms, AChR-ab positivity, and RNST results are not different from those usually found in MG. ${ }^{4}$ In addition, clinical features that are specific to ALS after MG might not exist, and the region of ALS onset seems to vary, independently of previous myasthenia symptoms. ${ }^{4}$ Half $(n=2 / 4)$ of all cases with bulbar onset ALS have been reported to be ocular MG, and our case represents pure respiratory onset ALS after ocular MG. ${ }^{4}$ The onset interval between MG and ALS is variable, ranging from 3 months to 41 years. ${ }^{4,5}$ Therefore, when muscle weakness develops in regions different from those of prior MG or are aggravated or reappear without definite fatigability, associations with other diseases such as ALS should be considered and tested, by confirming UMN and LMN signs and symptoms of motor neuron diseases as well as by EMG. 


\section{REFERENCES}

1. Park S, Lee JY, Lim NG, Hong Y. Incidence and prevalence of myasthenia gravis in Korea: a population-based study using the National Health Insurance Claims Database. J Clin Neurol 2016;12:340-344.

2. Jun KY, Park J, Oh KW, Kim EM, Bae JS, Kim I, et al. Epidemiology of ALS in Korea using nationwide big data. J Neurol Neurosurg Psychiatry 2019;90:395-403.

3. del Mar Amador M, Vandenberghe N, Berhoune N, Camdessanché JP, Gronier S, Delmont E, et al. Unusual association of amyotrophic lateral sclerosis and myasthenia gravis: a dysregulation of the adaptive immune system? Neuromuscul Disord 2016;26:342-346.

4. Tai H, Cui L, Guan Y, Liu M, Li X, Huang Y, et al. Amyotrophic lateral sclerosis and myasthenia gravis overlap syndrome: a review of two cases and the associated literature. Front neurol 2017;8:218.

5. Yamashita S, Fujimoto A, Mori Y, Hirahara T, Mori A, Hirano T, et al. Coexistence of amyotrophic lateral sclerosis and myasthenia gravis. J Neuromuscul Dis 2014;1:111-115.

6. Turner MR, Goldacre R, Ramagopalan S, Talbot K, Goldacre MJ. Autoimmune disease preceding amyotrophic lateral sclerosis: an epidemiologic study. Neurology 2013;81:1222-1225.

7. Ludolph A, Drory V, Hardiman O, Nakano I, Ravits J, RobberechtW, et al. A revision of the El Escorial criteria-2015. Amyotroph Lateral Scler Frontotemporal Degener 2015;16:291-292.

8. Gilhus NE, Verschuuren JJ. Myasthenia gravis: subgroup classification and therapeutic strategies. Lancet Neurol 2015;14:10231036.

9. Ohta K, Shigemoto K, Kubo S, Maruyama N, Abe Y, Ueda N, et al. MuSK antibodies in AChR Ab-seropositive MG vs AChR Ab-seronegative MG. Neurology 2004;62:2132-2133.

10. Rivner MH, Liu S, Quarles B, Fleenor B, Shen C, Pan J, et al. Agrin and low-density lipoprotein-related receptor protein 4 antibodies in amyotrophic lateral sclerosis patients. Muscle nerve 2017:55:430-432. 